\title{
Tangence
}

\section{Apocalypse sur l'écran (cathodique) : le crépuscule des dieux, version Updike}

Jean-François Chassay

Numéro 61, décembre 1999

Savoir et littérature

URI : https://id.erudit.org/iderudit/008167ar

DOI : https://doi.org/10.7202/008167ar

Aller au sommaire du numéro

Éditeur(s)

Presses de l'Université du Québec

ISSN

0226-9554 (imprimé)

1710-0305 (numérique)

Découvrir la revue

Citer cet article

Chassay, J.-F. (1999). Apocalypse sur l'écran (cathodique) : le crépuscule des

dieux, version Updike. Tangence, (61), 90-112. https://doi.org/10.7202/008167ar d'utilisation que vous pouvez consulter en ligne.

https://apropos.erudit.org/fr/usagers/politique-dutilisation/ 


\section{Apocalypse sur l'écran (cathodique): le crépuscule des dieux, version Updike Jean-François Chassay, Université du Québec à Montréal}

Soit Dieu est vivant, auquel cas il disposera de nous comme il l'entend. Soit il est mort, auquel cas il n'a jamais vécu vu qu'il y a peu de chances qu'il soit mort de vieillesse.

John Saul,

Le compagnon du doute

Il y aurait un "plan mathématique "du cosmos: rêve moteur, fructueux et irréalisable, objet idéal du désir.

Loup Verlet, La malle de Newton

La question du partage du savoir et du rôle de la connaissance en cette fin de $\mathrm{xx}^{\mathrm{e}}$ siècle, alors que triomphe le relativisme sous toutes ses formes, se révèle si complexe que l'aborder en détail dans un article aussi court serait une gageure intenable. À défaut de pouvoir y parvenir, je me contenterai ici de quelques propositions concernant la littérature, dans le contexte d'une réflexion sur les savoirs.

Il existe des hypothèses sur la littérature (des théories, si on veut), mais pas de science de la littérature. Quand les études littéraires se "scientifisent", c'est en passant par des domaines connexes (linguistique, pragmatique, sciences cognitives, etc.). Il ne s'agit pas d'un mode de connaissance au sens où ce mot s'entend généralement, selon un modèle hérité des sciences exactes au XIX $^{\mathrm{e}}$ siècle. On peut se demander cependant s'il n'en existe qu'un seul et s'il faut absolument des concepts et des modèles spécifiques, applicables sur commande. Au contraire, on pourrait avancer que la connaissance s'élabore par l'apport de concepts (ou même de postures critiques) provenant de différents horizons, interrogeant le sens de la réalité qui nous entoure et les événements qui s'y produisent. 
La science cherche à apporter des réponses, mais dans un univers social néo-libéral obsédé par la rapidité et l'efficacité, on oublie souvent de poser au préalable les questions. On peut même aller jusqu'à dire qu'on oublie parfois qu'il en faut, tant les dogmes sont puissants et résistent à celles-ci. Dans ce contexte, on comprend qu'il puisse être difficile de défendre l'idée que non seulement il existe des questions sans réponses, mais qu'il est essentiel de les poser et de les reposer, en espérant que certaines réponses toutes faites, certains automatismes culturels, vont parvenir grâce à elles à être ébranlés.

En ce sens, la fiction peut être vue comme le lieu d'un apprentissage du doute, du soupçon, du scepticisme, davantage que dans d'autres disciplines, dans d'autres univers discursifs - ce qui ne signifie évidemment pas que cela lui soit exclusif. La fiction est un mode d'apprentissage de la connaissance et non pas des connaissances: elle n'a pas de visée didactique, ne cherche pas à apporter des connaissances précises ${ }^{1}$. Sa fonction heuristique s'avère intéressante, pas sa fonction pédagogique - et on pourrait ajouter, à la limite, pas sa fonction logique (même si la cohérence d'un texte implique une logique interne). C'est un mode de compréhension du monde, pas un mode d'apprentissage de techniques particulières. La forme (les formes) qu'elle prend tend davantage à un mode d'analyse qui fonctionne par connexions, par analogies, par métaphores, plutôt que de reposer sur un rationalisme conceptuel. Cela n'implique pas une quête de sens moins grande qu'en philosophie, en sociologie ou en physique quantique.

Cette quête du sens, si elle prend dans la littérature des voies infinies, passe souvent depuis les origines du roman par une interrogation sur la science comme vecteur de connaissance. N'ayant pas pour raison d'être de présenter des résultats, le roman cherche plutôt à montrer comment la science se fait, quels dilemmes les scientifiques doivent affronter, quelles sont les apories qu'ils cherchent à masquer et, peut-être surtout, à travers quel langage ils expriment le monde qu'ils cherchent à objectiver.

Ce que pensait Roger, roman de John Updike, interroge les limites des sciences en les confrontant à la religion, opposant la

1. Alain Viala, de son côté, écrit que "la littérature est une réalité qui n'est pas unifiée". Georges Molinié et Alain Viala, Approches de la réception, Paris, Presses universitaires de France, 1993, p. 146. 
réalité des faits ${ }^{2}$ aux zones grises où notre savoir risque de déraper, incapable de résoudre certains problèmes fondamentaux ayant une valeur ontologique. Encyclopédiste, l'auteur ne lésine pas sur les disciplines convoquées. Ce que pensait Roger risque de perdre parfois le néophyte peu instruit en courbure spatiale et pour qui l'antimatière reste un mystère aussi impénétrable que celui de l'existence des Églises marcionites dans la Syrie du XVII ${ }^{\mathrm{e}}$ siècle. Mais dans ce roman, Updike parvient à faire oublier la thèse lorsqu'elle risque de s'alourdir. Par une série de glissements contrôlés, l'auteur passe de la théorie des quanta aux événements les plus prosaïques. Tout se retrouve sur le même plan et l'intérêt tient aussi à ce télescopage constant de l'anodin et du grandiose. Entre les discussions sur la conscience et la description d'une décharge urbaine, il ne semble pas y avoir de hiérarchie. Une même activité plutôt, une même volonté de voir et de penser le monde où convergent différentes manières d'interroger le sens de la fin.

Le décor de Ce que pensait Roger rappelle d'autres romans d'Updike. Le narrateur, Roger Lambert, 53 ans, travaille dans une petite ville universitaire de la Nouvelle-Angleterre. Ancien pasteur, il est devenu professeur de théologie après avoir laissé sa première femme pour sa maîtresse, Esther, avec qui il vit depuis maintenant 14 ans $^{3}$. La vie de Lambert prend une nouvelle tangente lors de la visite de Dale Kohler, étudiant en informatique de 28 ans. Grâce à lui, il fera la rencontre de sa nièce Verna (fille de sa demi-sœur Edna dont il n'a plus de nouvelles depuis des lustres) qui habite près de chez lui. Mais Kohler veut surtout son appui pour obtenir une bourse de la faculté. Il désire prouver l'existence de Dieu par l'informatique en le faisant apparaître sur l'écran et démontrer par ses calculs qu'il a vraiment créé l'univers. La ferveur du jeune scientifique sera contrée par le scepticisme du théologien:

Pour ma part, je dois avouer que, tant sur le plan esthétique qu'éthique, votre idée me paraît parfaitement répugnante. Esthétiquement, elle décrit un Dieu Qui intellectuellement Se

2. "[Les faits] sont devenus le réconfort et le pilier des idées reçues [...]. Des armées confuses de faits contradictoires s'affrontent dans une obscurité toujours plus grande. Servent à étayer des fantasmes idéologiques. Remplissent les dossiers préparatoires des bureaucrates." John Saul, Le compagnon du doute, Paris, Payot, 1996, p. 151.

3. Depuis Couples en 1968, Updike est devenu, si on peut s'exprimer ainsi, un spécialiste de l'adultère. 
laisse piéger, et éthiquement elle élimine la foi de la religion, elle nous prive de notre liberté de croire ou de douter. S'il existe un Dieu que l'on pourrait prouver, toute la chose en devient immensément, disons, inintéressante. ${ }^{4}$

Esther aura une liaison avec Dale pendant que Roger vit une relation conflictuelle avec sa nièce. Cette jeune femme qui vit seule avec un enfant de 18 mois - comme le dit suavement l'ancien pasteur: en apprenant la nouvelle, son "père, en chrétien convaincu, lui avait tourné le dos" (p. 345) —, incitera son oncle à la séduire et il ne pourra résister à la tentation de faire l'amour une fois avec elle. À la fin, Lambert et sa femme adopteront l'enfant de Verna pendant que Dale Kohler, de son côté, abandonnera un peu dépité son projet "sacrilège".

Le roman se déroule à l'époque de la réélection de Reagan alors que patriotisme se conjugue avec messianisme (le président ne s'opposa-t-il pas aux Soviétiques en parlant de "l'empire du mal?"). Il s'agit d'un moment clé au plan des transformations des valeurs. Les années quatre-vingt rapprochent singulièrement la population de la fin du millénaire, avec ce que cela suppose de fantasmes apocalyptiques, en même temps qu'elles amorcent l'ère de la virtualité avec la multiplication des réseaux et des possibilités de l'informatique, à la portée de tous. Cette époque de fin des certitudes - disparition des grands blocs sur le plan politique, critique radicale des "canons" esthétiques et moraux, remise en question de l'idée d'objectivité, même en science, etc. - conduit à un éclatement de l'homogénéité culturelle (et à la venue, dans une certaine mesure, d'une nouvelle Renaissance). Cela provoque en réaction une multiplication des Vérités et des Croyances reposant sur la nécessité ou la crainte de la Fin. De ce point de vue, la postmodernité, dans son acception sociologique, comme période postindustrielle conduisant à l'ère de la virtualité, serait aussi l'ère d'un fort retour du religieux. Dans le pays qui a vu naître l'ordinateur, 69\% des gens, selon une enquête de 1994, croient aux anges ${ }^{5}$. Est-ce une étrange relation de cause à effet? Quand l'impalpable devient la nouvelle réalité et la virtualité un

4. John Updike, Ce que pensait Roger, Paris, Gallimard, coll. "Du monde entier ", 1988, p. 38. L'édition originale américaine date de 1986. Toutes les références renverront à cette édition française.

5. Et selon un sondage récent du National Enquirer, $17 \%$ de la population croit qu'Elvis Presley est encore vivant. On peut se demander quel est le pourcentage de ceux-ci qui croient aux anges. 
94

phénomène normal, certaines personnes peuvent bien se mettre à croire justement à l'existence de ce qui n'existe pas. Cela donne des sectes, comme Heaven's Gate, où les gens se tuent sans conséquence: ils se contentent de partir pour une planète éloignée et se paient le luxe de l'annoncer sur internet.

Dale Kohler apparaît dans ce contexte comme un symptôme (pathétique) de ces individus assez au fait de la science pour s'en servir contre elle. Utiliser l'informatique pour découvrir Dieu s'avère en réalité une étrange double contrainte, propre peut-être aux fantasmes d'une fin de millénaire. Si Kohler réussissait, cela prouverait à la fois la mort de Dieu - que vaut un Dieu capable de se faire piéger par les petits calculs de ceux qu'Il a créés? et la mort de la science, obligé d'admettre l'existence de Celui qu'elle a toujours cherché à refouler, jusqu'à son anéantissement.

Mort de Dieu, mort de la science: voilà le risque pris par Kohler, à une époque où les paramètres épistémologiques se voient bouleversés. Mais les périodes de grands bouleversements ne révèlent-elles pas également de grandes craintes? Il faut revenir à ce sujet sur une conception un peu trop convenue de la Renaissance comme époque de la libération de la "grande noirceur "du Moyen Âge.

Les prophéties apocalyptiques [...] étaient tout à fait familières aux contemporains. Cette époque, qui fut marquée par tant de découvertes et de conquêtes, n'eut pour ainsi dire jamais le sentiment qu'elle voyait poindre l'aube d'un temps nouveau. Obsédée par la hantise du déclin, du péché et du Jugement, elle eut, au contraire, la certitude qu'elle était le point d'aboutissement de l'Histoire. ${ }^{6}$

La crainte de la fin, d'une apocalypse définitive au crépuscule de ce $\mathrm{xx}^{\mathrm{e}}$ siècle, comme à la Renaissance, s'inscrit dans Ce que pensait Roger sur tous les plans. Elle se manifeste à travers l'apocalypse qui guette la planète (à cause des envies martiales des politiques ou dans l'esprit de certains mystiques), les réflexions de Roger sur sa propre fin biologique, la fin de l'histoire, de Dieu, ou même de la littérature, comme on le constatera en conclusion en examinant brièvement les rapports intertextuels entre ce roman et l'œuvre fondatrice d'Hawthorne, La lettre écarlate.

6. Jean Delumeau (citant J. Lebeau), La peur en Occident, Paris, Fayard, coll. "Pluriel", 1978, p. 277. 
Le savoir de la fin est en étroite relation avec la fonction même du roman, toujours prêt à ruser avec la mort, usant de la raison pour mieux la conduire aux confins de l'irrationnel et de l'indicible. Bref, de tout ce qui échappe justement aux mots, dans la brèche ouverte par la perte du sens, de l'identité, de ce qui refoule le connu et le commun.

Le roman s'ouvre ironiquement sur des propos du narrateur annonçant qu'il travaille sur un monde...fini, et de plus dans un cadre suranné :

J'ai été heureux à la faculté de Théologie. Les horaires sont supportables, le cadre élégant, mes collègues inoffensifs et spirituels, accoutumés qu'ils sont aux ombres. Maîtriser quelques langues mortes, exposer en épisodes séquentiels l'histoire opiniâtrement énigmatique du christianisme primitif devant des classes remplies d'optimistes, de crédules et de dociles - il y a des moyens plus frauduleux de gagner sa vie. (p. 13)

Lambert se penche sur un monde qui n'existe plus et parle même de ce travail au passé comme s'il avait quitté la faculté, ce que rien dans le roman n'annonce. Qu'est-ce qui rapproche cet univers d'érudition un peu poussiéreuse et les derniers développements de l'informatique? Rien de moins que Dieu et, à travers une interrogation sur Celui-ci, une réflexion sur le point de départ et le possible point d'arrivée de la vie.

\section{Sur le langage de la science}

Kohler sous-entend par son discours - ce que Lambert ne comprend pas parce que son interlocuteur n'ose l'exprimer ou refuse de le voir - que Dieu et l'ordinateur forment une même entité, véritable synecdoque. Il est l'ordinateur. Celui-ci contient Dieu, en propose l'expression actuelle. Comme Lui, il se trouve partout. L'affirmation semble outrée, mais rejoint pourtant des propos de laudateurs de l'informatique jouant allègrement de l'hyperbole: "C'est face au modèle de l'ordinateur qu'il faut renouveler nos interrogations sur le devenir et la vie. C'est sous la lumière imprévue de l'intelligence artificielle que nous devons repenser la pensée. " ${ }^{7}$ Et plus loin:

7. Pierre Lévy, La machine univers, Paris, La Découverte, 1987, p. 7-8. 
Les systèmes de traitement de l'information effectuent la médiation pratique de nos interactions avec l'univers. Lunettes autant que spectacle, nouvelle peau régissant nos rapports avec l'environnement, le vaste réseau de traitement et de circulation de l'information qui buissonne et se ramifie tous les jours davantage dessine peu à peu la figure d'un réel sans précédent. Voici la dimension transcendantale de l'informatique. ${ }^{8}$

Devant pareille extase scientifique, on pense aux mystiques, Dale Kohler semblant réussir cette jonction. Dans "le cube", bâtiment où se trouve le centre informatique, n'a-t-il pas le sentiment de vibrer "au milieu d'un gigantesque bourdonnement qui évoque une activité spirituelle" (p. 279)? Placé devant un écran et pouvant utiliser un programme d'ordinateur aux ressources insoupçonnées, Saint Jérôme et Saint Augustin auraient-ils pu faire preuve d'une foi plus profonde?

L'univers, sous une forme stylisée et spécimen, existe au bout de ses doigts. Une crainte religieuse, ou la peur, l'effleure tandis qu'hésitent ses mains. Il n'a aucun dessein, aucun programme de manipulations pour aboutir au résultat final énoncé dans le titre prométhéen de son programme; il procède par la foi, se fiant à sa pieuse intuition pour le guider toujours plus avant au sein de ce labyrinthe forgé pour reproduire, dans l'essentiel, la réalité créée (aurait-elle pu ne pas l'être, créée?) (p. 285)

La réflexion sur la foi passe donc à travers la science et oppose paradoxalement un théologien sceptique à un scientifique profondément croyant. Les débats - essentiellement entre Lambert et Kohler, mais pas uniquement — obligent à argumenter sur les théories scientifiques.

Ceci a l'intérêt, d'une part, de présenter une science en train de se penser et non pas uniquement des résultats, d'autre part de montrer comment elle peut être confrontée à d'autres types de savoir et défendre, par le langage, ses propres arguments (parfois de manière subjective et passionnée, ce qui se révèle en soi passionnant). On affirme souvent que plus la science s'avère "sérieuse" (près de la vérité...), plus elle s'énonce par un grand formalisme, ce qui lui permettrait d'échapper au langage argumentatif dont la subjectivité serait aux antipodes d'une "vraie" pensée scientifique. Ainsi, la physique théorique aujourd'hui serait la reine des sciences et la mathématique son plus sérieux

8. Ibid., p. 10. 
challenger. Pourtant, comme le souligne Jean-Marc Lévy-Leblond, "le niveau de formalisation d'un discours, quel qu'il soit, ne saurait être considéré comme un critère de scientificité - il faudrait, sinon, tenir les partitions musicales pour les plus anciens des textes scientifiques." 9 De plus,

[n]ombre des problèmes conceptuels qui continuent à hanter la physique contemporaine (la question du déterminisme, ou celle de l'origine de l'univers) trouvent leur source dans la négligence des puissances de la langue et dans la désinvolture de son usage; le montrent le consensus assez général sur les formalismes mathématiques, justement, et le désaccord sur leur interprétation: c'est bien que la formalisation ne suffit en rien à énoncer et à maîtriser le savoir qu'elle propose. ${ }^{10}$

Lors d'une discussion avec un certain Kriegman à propos des origines de l'univers, Kohler relève qu'il parle de "poussière de points", "gèle", "graine" — tout ça ce sont des métaphores". À cela Kriegman répond: "Existe-t-il autre chose? [...] Comme dit Platon: des ombres tapies au fond de la caverne" - ce qui ne l'empêche pas d'ajouter: "Pourtant, impossible de se passer de la raison" (p. 365).

Enfin, comme le rappelle Bruno Latour et Steve Woolgar ${ }^{11}$ à plusieurs reprises dans leur livre, le travail de laboratoire est un échange, et un échange parlé, ce que confirme Lévy-Leblond:

Le chercheur - a-t-on assez dit dans quel état de frustration permanente il travaille? - se heurte donc sans trêve à ses propres limites. Rien de plus naturel alors que le miroir de l'autre. C'est l'une des pratiques spontanées du chercheur, quand il piétine devant une difficulté technique de son montage ou une erreur masquée de son calcul, que d'aller exposer son problème au collègue voisin - lui en parler: la sortie du bureau est l'issue de secours (en tout cas, l'issue de recours) naturelle. ${ }^{12}$

L'invention des idées forme un processus qui fonctionne à la fois grâce à l'intuition, à l'imagination, à l'expérience, à la

9. Jean-Marc Lévy-Leblond, La pierre de touche, Paris, Gallimard, coll. "Folio inédits", 1996, p. 234.

10. Ibid., p. 233.

11. Bruno Latour et Steve Woolgar, La vie de laboratoire. La production des faits scientifiques, Paris, La Découverte, "Poche", 1996, 299 p.

12. Jean-Marc Lévy-Leblond, op. cit., p. 253-254. 
98

connaissance et au désir, ceci étant indissociable du contexte discursif dans lequel baigne l'individu. Un roman comme Ce que pensait Roger montre comment les idées viennent, comment elles se forment et comment on en vient à formuler certaines hypothèses. Ceci dans un texte où les dialogues occupent une grande place. Platon est là pour rappeler qu'ils sont la forme même de toute pensée qui se fait. Mais il est également bon dans ce contexte de souligner, à la suite de Jean-Marc Lévy-Leblond encore une fois, que

[1]'écrit fondateur de la physique au sens moderne, celui qui inaugure véritablement la science telle que nous la connaissons, s'intitule Dialogues (sur les deux grands systèmes du monde) [...]. L'utilisation de la langue commune et le recours à la forme la plus achevée de la rhétorique (depuis Platon) font ici le génie de Galilée, non moins que, et inséparablement de, l'importance et la nouveauté de ses idées. ${ }^{13}$

"Le dialogue est un mode effectif de la science en acte [...] Ce n'est donc pas une simple coïncidence si [...] la science contemporaine s'inaugure dans le dialogue de Galilée. La force de conviction rhétorique (le travail de propagande, dirait Feyerabend) de ce texte vient précisément de ce qu'il explicite le violent affrontement dont il participe. " 14 On pourrait ajouter, pour citer cette fois Calvino, que Galilée "utilise le langage, non pas comme un instrument neutre, mais avec une précision littéraire, avec une continuelle participation expressive, imaginative, et même lyrique [...]. Le regard sur le monde du Galilée scientifique est nourri de culture littéraire " 15 .

Mais le roman d'Updike ne consiste pas seulement en un dialogue sur la science. Il s'inscrit dans un contexte discursif où la réalité sociopolitique se mêle à l'intime, où l'enracinement culturel devient indissociable de l'Histoire. Les réflexions nombreuses autour du discours scientifique s'articulent aux problèmes subjectifs d'individus aux prises avec le réel, avec des fins personnelles se surimposant à la fin (aux fins) collective(s).

13. Ibid., p. 256.

14. Ibid., p. 263.

15. Italo Calvino, La machine littérature, Paris, Seuil, 1984, p. 33. 


\section{La guerre des chiffres}

La science dans Ce que pensait Roger s'articule pour l'essentiel autour de trois séries d'arguments qui se croisent, liées à trois grands mythes ou récits scientifiques: la symbolique des chiffres, associée ici à l'ordinateur; la théorie de l'évolution; l'origine de l'univers. Je voudrais m'y arrêter pour voir comment ils se conjuguent pour mettre en lumière un imaginaire de la fin mêlant science et théologie.

On associe généralement l'apocalypse à la catastrophe et à la fin du monde. Cette interprétation, recevable, n'est pas la seule possible. Apocalypse signifie également "révélation", "dévoilement", soulever le voile pour découvrir ce qui se cache. "Révélation de qui à qui? D'une entité divine à un être humain. Dévoilement de quoi? De secrets que nul homme ne connaît, secrets proprement divins que Dieu choisit de révéler à l'un de Ses élus, homme au cour pur, qui sera messager parmi les autres hommes, transmetteur de ces connaissances nouvellement reçues." ${ }^{16}$ Cette apocalypse ressemble à celle de Kohler, une quête qui dans son cas passe par des chiffres. Il faut noter cependant qu'il n'est pas le premier: au XVII ${ }^{\mathrm{e}}$ siècle, le mathématicien anglais John Neper inventa les logarithmes pour calculer au plus près la date de la fin du monde. Mais les possibilités de l'ordinateur n'ont pas de commune mesure avec ce que la mathématique pouvait offrir il y a 300 ans, comme l'explique Kohler à sa façon:

Il existe maintenant une nouvelle branche de mathématiques, à mi-chemin des maths et de la physique, en fait il est indispensable d'utiliser l'ordinateur [...]. Mon impression personnelle, c'est qu'avec ce type de comportement mathématique, on se rapproche énormément de la texture même de la Création, si l'on peut dire [...] Voilà à quoi commencent à servir les ordinateurs, à cette étude du chaos, de la complexité. Les implications sont énormes; s'il est possible de modeler l'univers physique grâce à un système de calcul et de considérer ses lois comme des algorithmes, dans ce cas, [...] il serait possible de modeler la réalité elle-même, et ensuite de l'interroger! (p. 129130)

Pour Kohler, d'un point de vue rationnel (et là se trouve l'intérêt de son argumentation), l'univers ne peut avoir été créé

16. Claude Kappler, "Apocalypses", Magazine littéraire, no 232, 1986, p. 16 (Les écrivains de la fin du monde). 
sans avoir été pensé par un esprit supérieur, les hasards étant trop nombreux. Dans cette perspective, la récurrence de chiffres "prouve" selon lui la présence de Dieu et rappelle par ailleurs l'Apocalypse selon Saint Jean: le 7 (comme pour les anges, les têtes, les chandeliers, les étoiles, les plaies), le 4 (les animaux), les 42 mois, etc.

La scène au cours de laquelle Kohler traque de manière obsessionnelle ses 2 et ses 4 - "[les] 2 ou [les] 4 [...], il s'en est quasiment convaincu, sont les chiffres sacrés par le truchement desquels Dieu s'adressera à lui " (p. 288) - survient au moment où une commission universitaire du Département de théologie le reçoit pour décider si oui ou non elle lui accordera une bourse.

[I]l y avait ces fichus vingt-quatre qui me sautaient aux yeux. Le temps de Planck, par exemple, divisé par la constante du rayonnement, donne un chiffre pratiquement équivalent encore une fois à huit dix puissance moins vingt-quatre, et la permittivité du vide [...] dans le rayon de Bohr donne presque exactement six dix puissance moins vingt-quatre. Côté puissances positives, la constante structure fine électromagnétique multipliée par le rayon de Hubble [...] donne approximativement dix puissance plus vingt-quatre et la constante interaction forte multipliée par la charge qui s'exerce sur le proton donne exactement deux virgule quatre dix puissance moins dix-huit. Chaque fois que vingt-quatre apparaissait sur le listing, j'ai commencé à l'entourer d'un trait: tenez - il le brandit, son morceau de tapisserie zébré d'innombrables rayures, orné d'innombrables cercles rouge vif —, facile de voir que ça n'a rien d'une simple contingence. [...] Dans ces mains, il tenait l'univers. (p. 258-259)

Le scepticisme des membres de la commission est palpable, qui font remarquer que les chiffres se manipulent aisément. Alors que Kohler répond affirmativement à une question portant sur un calcul donnant plusieurs six consécutifs, il se fait dire par un membre de la commission: "Vous voyez [...] Eh bien, il s'agit là du chiffre du Diable, et en principe ce chiffre signifie que la fin du monde est proche" (p. 260). Un autre ajoute: "Ou que deux est en train d'être divisé par trois [...] Pour moi ces calculs, jeune homme, ont une certaine saveur de désespoir" (p. 260).

Que le seul vrai débat sur les nombres survienne au moment du passage de l'étudiant devant une commission qui ressemble à une inquisition ne manque pas de sel, puisque le but de celle-ci 
était de juger les hérétiques. Étudier ceux qui s'écartent de la doctrine de la foi chrétienne constitue justement la spécialité de Lambert et le pont sera sans cesse franchi entre Kohler et ces hérétiques du Ier siècle qui annonçaient la fin du monde.

Cela constitue une spécialité de l'universitaire non seulement à cause de ses intérêts théorique et historique, mais aussi à cause de son statut de professeur de théologie aux États-Unis lui permettant d'observer "l'actuelle génération de fanas de Jésus [qui] affichent une mansuétude amorphe de zombies et une innocence historique irréductible qui tendent à me rendre fou de rage" (p. 20-21). À Kohler, il dira: "Vous êtes tous des fanatiques de l'amour, du mot amour, sinon de la réalité qu'il recouvre" (p. 30) avant d'ajouter: "Les dévots ont souvent, je l'ai remarqué, un côté catégorique qu'ils jugeraient grossier chez les autres" (p. 31). Voilà qui, sur le mode mineur, lui rappelle les hérétiques comme Marcion dont il peut se vanter d'être un expert: ceux qui savent la vérité, affirment savoir comment les choses se sont passées et ce qu'il faudrait faire pour éviter la catastrophe ultime. Il multiplie ainsi, directement ou indirectement, les comparaisons sur ce plan avec l'époque moderne et le fanatisme des étudiants comme Dale Kohler: "la flamme missionnaire, la volonté de convertir, de transformer l'eau en vin, le vin en sang, le pain en chair - de convertir l'opposition en obéissance, d'aplatir tout ce qui est nonego pour le réduire en ego lisse comme un miroir. Au fil des années, cette éternelle présomption des étudiants me lasse et me répugne" (p. 112).

Au-delà des individus, cela signale un état du discours social qui permet à Lambert de répondre à Kohler quand celui-ci lui parle des miracles "attestés"par la Bible. Après avoir évoqué le sens très différent de l'écriture il y a 2000 ans ("Écrire était une forme de magie par transfert, ne l'oublions pas", p. 108), il indique la quasi-impossibilité d'interpréter de manière juste:

Quand on ne connaît pas et ne peut espérer jamais connaître les tenants et aboutissants exacts d'un événement ou d'un phénomène, une certaine intuition est tout ce que l'on peut suivre pour juger de ce qui est plausible. Quand je lis le National Enquirer, que je vois toutes ses histoires de petits hommes verts décrits dans les moindres détails qui émergent des ovnis, ou la dernière preuve irréfutable qu'Elvis est toujours vivant, il me vient une vague intuition de ce qui s'est peut-être passé tout au début du premier siècle. (p. 109) 
102

Si les rapports entre Lambert et Kohler ressemblent à un dialogue de sourds, celui-ci ne tient pas seulement au fond mais également à la forme. Et cela ne vaut pas uniquement pour ces deux personnages. De la réflexion sur les systèmes autoreproducteurs à la théorie du Big Bang, des exégètes de la Bible à l'intelligence artificielle, le discours fait souvent preuve d'une réelle opacité. Dans le corps du texte se multiplient les explications et les affirmations sur des phénomènes que la plupart des lecteurs ne peuvent juger dans le détail. Dans le contexte d'un roman où la Bible sert souvent de référence, le lecteur est renvoyé à une nouvelle tour de Babel.

L'informatique est née de la cybernétique, cette science des communications et de la régulation dans l'être vivant et la machine, apparue dans les années quarante. Son objectif premier était de fournir un métalangage commun à diverses disciplines scientifiques pour permettre à des gens ouvrant dans des champs de plus en plus spécialisés de pouvoir travailler en commun. La volonté d'interdisciplinarité favorisée alors éclate dans le roman d'Updike. C'est comme si Dieu, que Kohler veut faire apparaittre sur l'écran de l'ordinateur (à son image) punissait ceux qui, comme à l'époque de Babel, veulent se rendre jusqu'à lui.

Au cour des années quatre-vingt, le roman donne l'impression exactement inverse de ce que voulait produire la cybernétique: le langage, crypté, rend difficile les interactions et l'importance des sectes, omniprésentes tout au long de la narration, se trouve à surgir ironiquement au sein même du discours scientifique. L'opacité du langage va de pair avec les réflexions sur l'obscurantisme, notamment sur le créationnisme dont il sera question plus loin. Mais cette opacité annonce aussi la fin du discours, l'impossibilité de se comprendre vraiment à l'ère des hyper-spécialisations. Elle tient également à la dimension indicible de ce qui cherche à être abordé. Plus on cherche à approcher de la Vérité, plus les mots paraissent inadéquats. Aux blancs, aux silences - nous ne sommes ni chez Blanchot ni chez Beckett —, Updike substitue la logorrhée de discours qui ressemblent à des dialectes. "Jadis variations du langage dues à l'isolement géographique, les dialectes sont désormais des variations encouragées par les spécialistes pour empêcher l'accès des noninitiés à leur territoire professionnel. " 17 Un fil conducteur

17. John Saul, op. cit., p. 118. 
pourtant permet de rapprocher les discours, de la plus haute abstraction à la plus banale trivialité : l'évolution et ses affres.

\section{Dégénérescence et évolution}

L'isotopie évolutionniste allie les aléas de la vie sexuelle de Roger à la critique du darwinisme, en passant par l'état de la politique américaine sous Reagan. Elle permet également de manifester des failles épistémologiques dans l'argumentation de Kohler. On sait que la théorie de l'évolution ne date pas de Darwin. Les thèses de Lamarck naissent au début du XIX ${ }^{\mathrm{e}}$ siècle et sont alors bien reçues. Le scandale de L'origine des espèces, en 1859, repose sur les mécanismes de l'évolution, à savoir la sélection naturelle. Cela suppose qu'il n'existe plus de maître de jeu de l'évolution, Dieu ou raison, mais simplement le hasard. L'évolution darwinienne n'a pas de finalité morale. Un des chemins mène à notre espèce, mais cette bifurcation est accidentelle.

La théorie de l'évolution, en ce $\mathrm{xIx}^{\mathrm{e}}$ siècle où le progrès resplendissait de tous ses feux, a donné lieu à une fiction (commode) selon laquelle l'homme descend du singe, thèse attribuée à tort à Darwin (qui affirmait plutôt que l'homme en est un cousin éloigné). L'ancêtre de l'être humain n'a pas été créé à la suite d'un projet grâce à l'étincelle divine mais par accident, il y a 5 millions d'années, à l'emplacement du Kenya actuel. L'australopithèque, premier "hominien" connu, a conduit, par sélections ultérieures, à l'Homo Sapiens, apparu il y a 200000 ans à peine (sur une planète qui a environ 4 milliards 500000 millions d'années) ${ }^{18}$. En modifiant la pensée de Darwin, on propose d'une part une vision linéaire de l'évolution qui ne tient pas, et d'autre part on fait de la complexité humaine un aboutissement sans plus de crédibilité.

Il est fréquent de confondre progrès et évolution, transformant le premier terme en adjectif au service du second (une évolution progressive). Or, cette conception simpliste est fausse, ne correspond pas aux propos de Darwin et des néo-darwinistes. C'est un des problèmes de Kohler pour qui l'aléatoire ne peut se lire qu'à l'aune d'un système téléologique. Le temps reste une

18. La production sur ces questions est immense, mais les ouvrages de Stephen Jay Gould restent des mines de renseignements et de plaisir littéraire. 
104

donnée essentielle de l'évolution. Avec quelques millions d'années, le hasard peut provoquer une infinité de combinaisons. Pour Kohler, qui tente de le démontrer exemples à l'appui, le hasard ne peut pas être si hasardeux que ça.

La théorie de l'évolution trace un portrait dévalorisant de l'être humain, évacué du centre de la création et annonce ainsi, à défaut de la fin du monde, la fin d'un monde. L'Homme n'est plus immortel mais un simple rameau secondaire dans l'évolution, l'origine ultime se conçoit difficilement, l'univers tend vers une entropie maximale présageant sa fin (lointaine, ce qui ne change rien au problème). De l'eugénisme de Francis Galton jusqu'au nazisme (de manière incommensurablement plus tragique dans ce dernier cas), cette peur de la perte des repères a conduit à deux doigts d'une réelle catastrophe finale.

La situation ne manque pas d'être délicate. Plus les sciences se développent, plus elles conduisent au désenchantement et à la désillusion. On en voit des traces chez Updike, que ce soit lorsque Lambert pense au cancer et à la mort parce qu'il fume une pipe ou, plus banalement encore, lorsqu'il goûte à un vin: "Bien sûr, la fermentation est une forme de pourriture, exactement comme la vie, considérée sous l'angle de l'énergie, est une forme de décrépitude." (p. 61)

Kohler s'oppose à ce désenchantement et aux prétentions de la science qui n'en finit plus, selon lui, de colmater des brèches méthodologiques pour expliquer et justifier le monde à sa façon, s'embourbant pour mieux masquer ses failles dans un langage abscons et hyper-spécialisé - manière de discourir qu'on pourrait d'ailleurs ironiquement lui attribuer. Il attaque la théorie de l'évolution en s'appuyant sur un développement linéaire que les darwinistes récusent pourtant. Mais en cherchant à démolir les émules de Darwin, Kohler cherche aussi à évacuer les vertiges et les peurs qui se développent lorsque les balises disparaissent. Il affirme, parlant de l'évolution, que "c'est tellement bordélique" (p. 110). L'ordre émerge pourtant du chaos et semble "ordonné" parce qu'on s'y habitue. Il y a deux façons d'interpréter cette recherche d'un ordre supérieur.

D'abord, cette réflexion chaotique autour de l'évolution se dessine avec en arrière-plan les agissements d'un gouvernement républicain aux visées idéologiques très nettes. Kohler ne s'intéresse pas à la politique mais il est symptomatique que Lambert 
fasse allusion à Reagan dès leur première rencontre. Alors que l'étudiant avance que Verna, en refusant d'avorter, avait des motifs religieux, "le choix de ne pas tuer", Lambert réplique "Ainsi, vous êtes de la même école que notre président?" (p. 17). Les allusions à Reagan sont récurrentes sans être très nombreuses, mais rappellent l'aura mystique qui l'environne:

Tout au long de ce mois de décembre, le temps demeura chaud, comme si les cieux eussent voulu accorder leur bénédiction à notre choix national, notre réélection. Dieu resplendissait à travers le président, semblait-il à beaucoup, et aux yeux des autres, il était une force de la nature qu'il était vain de vouloir défier. Parmi ceux qui avait voté pour son rival, beaucoup se réjouissaient en secret de sa victoire; il demandait si peu, il promettait tant. (p. 160)

Et plus loin encore: "[...] il me semblait que nous existions tous à l'intérieur de la tête placide et creuse de Reagan comme à l'intérieur d'une bulle géante, et qu'un jour viendrait peut-être où cette bulle exploserait, et où ceux d'entre nous qui aurions survécu, repenserions à cette Amérique d'aujourd'hui comme à un paradis" (p. 350). Se pourrait-il que l'ordre rassurant que propose Reagan influence un homme apparemment aussi détaché des contingences politiques que Kohler?

Le nom de Reagan évoque également deux phénomènes qu'on peut lier à l'imaginaire de la fin. Le premier, social, touche le retour en force des créationnistes qui, forts de l'appui du candidat républicain, ont fait plus que jamais pression, au cours de la décennie, pour que les écoles américaines accordent autant de place à la théorie scientifique de l'évolution qu'au récit de la Genèse. Liés aux millénaristes, les créationnistes font partie de la droite fondamentaliste américaine qui voyait dans Reagan son champion (et qui a été déçue - les Républicains savent quand même être pragmatiques...).

Kohler ne peut être considéré comme un créationniste militant. Il s'agit plutôt d'un flipper, un computer hacker ayant donné une orientation particulière à son travail sur des systèmes informatiques hyper-puissants. Dévoiler Dieu grâce aux possibilités de l'ordinateur, il fallait pour y penser avoir une foi un peu particulière... Si Lambert mentionne peu les créationnistes, la présence de Kohler permet de rendre compte du très vaste spectre de la croyance aux États-Unis et de la variété des cataclysmes escomptés. 
106

Le deuxième phénomène doit se prendre au sens métaphorique et a souvent été utilisé à propos du "pote à Bonzo" (p. 365). Il s'agit des dinosaures. Il en est question au moins indirectement lorsque Kohler s'attaque au néodarwinisme: "Il n'y a presque rien, sinon des espèces entières qui apparaissent et disparaissent. Les pseudo-hiatus [parmi les espèces] ne sont pas des hiatus, ce sont des trous énormes, des trous monstrueux" (p. 102) ${ }^{19}$. À l'arrière-plan de ce débat souvent différé, reporté, sur l'évolution et sur Dieu, se profile cette figure du dinosaure, comme symbole idoine de l'énigme de l'évolution.

Si Reagan cependant apparaît comme un dinosaure, réactionnaire incapable de comprendre le progrès de son époque, Lambert en est également un, à sa manière. Qui aujourd'hui s'intéresse vraiment à Pélage? Aux églises marcionites et aux pamphlets antimarcionites d'Epiphane? Esther elle-même lui reproche d'être allé se spécialiser " dans ces gens abominables, ces fanatiques, ces fossiles dont il ne reste pratiquement rien, pas même la peau et les os" (p. 153). Voilà des fossiles qui rejoignent Reagan d'une autre manière et viennent boucler la boucle de l'évolution, proposant deux traditions différentes de dinosaures.

Si on rejette l'hypothèse selon laquelle la pensée de Kohler subirait l'influence de l'ère Reagan - et on pourrait dire que ce roman pose justement la question: qu'est-ce qu'une hypothèse? Comment proposer intelligemment une théorie? - une deuxième interprétation de cette recherche d'un ordre supérieur apparaît plausible, dont la portée est beaucoup plus universelle.

Kohler souffre de ne pas vivre sa petite apocalypse (pour reprendre le titre d'un roman de Konwicki) et espère désespérément avoir droit au dévoilement, à la preuve d'un ordre supérieur. "L'espèce humaine est seule parmi des millions d'autres espèces, et elle ne bénéficie d'aucune prévisibilité cosmique. Mais cela ne doit pas nous inquiéter. Le sens est un besoin humain, l'univers ne recherche pas le sens." ${ }^{20}$ Malgré ses tics et ses mau-

19. C'est vrai, mais cela n'a rien de tout à fait exceptionnel. Selon les paléontologistes de l'Université de Chicago, il y aurait eu cinq épisodes d'extinctions massives dans l'histoire du vivant et notamment les dinosaures qui auraient dominé la planète 100 millions d'années, soit 500 fois plus longtemps que l'Homo Sapiens jusqu'à maintenant.

20. "Nos ancêtres les bactéries. Entretien avec S. J. Gould", Le Nouvel Obs horssérie, 31, "La quête des origines", p. 57. 
vaises habitudes, l'agacement qu'il peut provoquer chez Lambert (et chez le lecteur!), cet aspect de sa personnalité reste touchant. Kohler vit intensément, de manière exacerbée, ce que chacun peut vivre, à divers moments de son existence: la difficulté à concevoir que le sens n'ait aucune valeur à l'échelle de l'univers. Le choc de la théorie de l'évolution, comme des recherches en astrophysique depuis plusieurs décennies, tient à cette rupture entre la conscience humaine et le monde qui l'entoure. Tout individu cherche, parfois malgré lui, un ordre, une cohérence, à la limite un "plan" qui puisse donner sens au réel. Le savoir scientifique imposant de Kohler, qu'il ne cesse d'utiliser tous azimuts pour convaincre, signale de manière spectaculaire que "l'accumulation des faits ne suffit pas à étouffer la question du sens " ${ }^{21}$ mais plutôt la prolonge.

Comme tous les gens qui n'admettent pas le hasard cependant, Kohler manque de patience. Sa méthode de travail le confirme. À la recherche d'une réponse à ses questions, il néglige les indices, ne fournit pas un vrai travail de détection, agit en mauvais sémiologue. En fonctionnant de manière linéaire, erreur épistémologique fondamentale, il commence par le début, rejetant sarcastiquement la théorie du Big Bang. Mais ce qui semble aller de soi pour la conscience humaine ne l'est pas nécessairement à l'échelle de l'univers.

\section{L'univers par la fin}

Dale Kohler cherche tout de suite l'origine pour expliquer le monde actuel et, tel qu'il présente la chose, cela ne manque pas de ridicule.

Tout au début, ces boniments faciles au sujet de la "soupe originelle", dans laquelle des éclairs mijotent des acides aminés, puis des protéines, et enfin une chaîne d'ADN autoreproductible à l'intérieur d'une sorte de bulle qui était en fait la première cellule, ou la première créature — ça paraît formidable, mais voilà, ça ne tient pas, c'est du même acabit que ces histoires de mouches et d'araignées surgies par génération spontanée du fumier, des meules de foin, bref partout où au Moyen Âge les gens croyaient dur comme fer que ça se passait. (p. 100)

21. Loup Verlet, La malle de Newton, Paris, Gallimard, coll. "Bibliothèque des sciences humaines", 1993, p. 13. 
108

On oublie souvent que l'expression de "Big Bang" (littéralement: le gros boum...) a été inventée par le Britannique Fred Hoyle pour ridiculiser cette théorie ${ }^{22}$. C'est dans cet esprit que se situe Kohler. Ses affirmations laissent par ailleurs croire que tout le monde s'entend sur l'origine de l'univers. Pourtant, l'idée que dans "une fraction ridiculement brève de la première seconde du Big Bang et sous l'effet de certaine force théorique antigravitationnelle que personne n'a jamais vu agir, l'univers s'est dilaté exponentiellement, doublant environ tous les dix puissance moins trente-quatre secondes environ" (p. 29) est loin d'emporter une large adhésion. L'étudiant renvoie ainsi la science à la mythologie.

D'après ce que l'on sait actuellement, l'univers aurait 15 milliards d'années ${ }^{23}$. Pendant 2500 ans, on a cru à un univers éternel et inchangé. Même Einstein au début des années cinquante y croyait encore. Depuis 1965, donc depuis à peine un peu plus de trente ans, on a pu confirmer la théorie de Gamov datant de 1948. L'univers, changeant, était très chaud à l'origine et il existe des traces de ce passé: des rayonnements électromagnétiques qui jouent le même rôle pour les astrophysiciens que les fossiles pour les paléontologues.

En trente ans à peine, le progrès sur la question se révèle immense, ce qui peut inciter à sauter indûment aux conclusions et traverser le mur du Big Bang. Ne faudrait-il pas plutôt, comme le suggère Reeves, faire preuve de plus de méthode en proposant une exploration vers le passé, reprenant le principe de la Terra Incognita sur les cartes des explorateurs de la Renaissance, dont la dimension diminuait au fil des découvertes?

Depuis six ou sept milliards d'années, l'univers pourrait se résumer par une croissance continue de sa complexité. Il a engendré de la structure, à toutes les échelles: galaxies, étoiles, planètes, comètes, animaux, molécules, atomes, électrons. Kriegman affirme à Kohler: "La persistance exige le temps, d'accord? Et là est la clef, précisément: faute de temps il n'existe rien" (p. 363). Pour ce dernier cependant, la complexité du

22. Voir Jean-Marc Lévy-Leblond, op. cit., p. 240-241.

23. Ce qui suit au cours des prochaines lignes est tiré d'un article d'Hubert Reeves, "L'origine de l'univers", Horizons philosophiques, vol. II, n⿳ 2 , printemps 1992, p. 1-26. 
processus ne se concilie pas avec sa conception du sens. Pourtant tout processus, comme celui de la pensée, est complexe, comporte une part d'aléatoire, des connexions qui peu à peu s'organisent pour former quelque chose. "Dans votre corps, quand vous faites une action, quand par exemple vous fermez les yeux et que vous dites: "j'existe”, ce simple geste demande l'entrée en opération de ces cent milliards de milliards de milliards de particules qui jouent chacune un rôle précis. Il est fondamental qu'elles le jouent et qu'elles le jouent bien, sinon vous ne serez pas en mesure de réfléchir. "24

Kohler refuse d'assumer le vertige provoqué par un tel "miracle" de la nature et préfère le justifier par une entité supérieure. Il doit y avoir une logique causale pour tout expliquer selon un processus linéaire. Dans un contexte différent, la position épistémologique de Kohler reste assez proche de celle de Lamarck. Il faut dire que ce scientifique a un étrange rapport à la science. Par exemple, il rejette l'idée qu'un ordinateur puisse penser, prenant le contre-pied d'un large courant cognitiviste: "Toutes ces histoires d'ordinateurs capables de penser, pour qui s'y connait, c'est un fantastique non-problème. On pourrait s'amuser à connecter tous les ordinateurs en service d'ici à Palo Alto, n'empêche [...], on ne pourrait obtenir la conscience: autant de chances de l'obtenir des fils et des standards du téléphone" (p. 203). Sa foi, il la transmet à (dans) la machine informatique. L'ordinateur ne peut avoir de conscience car il s'agit d'une machine simple. Ce qui manque de simplicité — par exemple la complexité des processus de l'évolution - ne peut pas être vrai. Alors que, paradoxalement, un miracle est simple. Donc, Dieu existe. Étrange syllogisme qui maintient la foi de Kohler. Pourtant, il propose de réaliser quelque chose de terriblement contre-nature.

Les créatures artificielles peuplent depuis toujours une zone particulière de notre imaginaire ${ }^{25}$. Le développement de l'intelligence artificielle, à partir des années cinquante, reprend cet antique mythe. Les cognitivistes soutenaient dès le départ le projet de construire un cerveau dont les performances seraient équivalentes ou supérieures à celles d'un cerveau humain et permettrait d'en faire une machine à l'image de l'Homme. C'est, pourrait-on dire à la suite de Philippe Breton, le récit fondateur de l'Intelligence

24. Ibid., p. 21.

25. Voir Philippe Breton, À l'image de l'Homme, Paris, Seuil, 1995. 
110

artificielle et de toutes les disciplines contemporaines qui lui sont connexes.

Il n'y a pas de créations artificielles dans Ce que pensait Roger mais on peut voir des traces inversées de ce récit fondateur. Et rien ne peut apparaître plus prétentieux que cette volonté de la part de la créature de créer le créateur lui-même. Même s'il ne l'exprime pas sur ce ton, Lambert y voit le pire des sacrilèges: après avoir fait l'Homme à son image ${ }^{26}$, voici que Dieu devrait supporter de voir l'Homme le reproduire. Dale Kohler se décourage cependant et ne permettra pas de répondre de manière "réaliste "à la question. Dieu existe-t-il? En vérité, Il a toujours une place dans le roman, et dans celui-ci de manière particulièrement significative. Dans Ce que pensait Roger, Lambert, ancien pasteur, joue lui-même de manière indéniable ce rôle, indiquant son pouvoir divin sur le plan narratif. Non seulement s'agit-il d'un narrateur autodiégétique mais bel et bien d'un narrateur omniscient au sens fort. Il raconte et voit tout, y compris ce qu'il ne peut logiquement qu'interpréter. Par exemple le travail de Dale au laboratoire, ses frustrations, ses interrogations, ses craintes. En outre, il "voit" les ébats d'Esther et de Dale. Non seulement est-il omniscient comme Dieu, mais le lecteur a affaire à un Dieu cocu! Il est même en droit de se demander si Lambert n'invente pas cette relation amoureuse. Il lui arrive, fatigué, de fermer les yeux et de "voir" — et par le fait même de décrire au lecteur, comme si on y était - Esther et Dale avoir des relations sexuelles. Rien, jamais, ne viendra confirmer ce qui est présenté par Lambert comme une évidence. Il reste jusqu'au bout, au sens fort, le créateur de cette histoire qui bien sûr aura une fin. Malheureuse.

\section{En guise de conclusion: la fin de la littérature (vieille antienne)}

Par ce clin d'œil qui place Dieu dans l'orbe du roman, Updike rappelle que l'univers littéraire a lui-même ses propres lois, ses propres règles avec lesquelles il lui est loisible de jouer. Je terminerai en rappelant très brièvement la dimension intertextuelle de ce roman qui propose un autre jeu sur la fin, qu'on pourra

26. Pour reprendre l'adage: "Dieu existe et elle est noire..." 
interpréter sérieusement ou avec le sourire, c'est selon (avec Updike la deuxième option semble généralement plus sûre).

Jean Delumeau écrit dans La peur en Occident que la grande période de peur eschatologique (et, à rebours, la peur de l'an Mille) date de la Renaissance et s'étend jusqu'au milieu du $\mathrm{XVII}^{\mathrm{e}}$ siècle. Rien ne peut l'exprimer plus clairement que ces propos du prédicateur Geiler, lancés en 1508 de la cathédrale de Strasbourg et qui se passent de commentaires: "Ce qu'il y a de mieux à faire, c'est de se tenir en son coin et se fourrer la tête dans un trou en s'attachant à suivre les commandements de Dieu et à pratiquer le bien pour gagner le salut éternel. "27

C'est dans ce contexte de peur qu'il faut situer l'arrivée des Puritains en terre américaine, l'accostage du Mayflower en 1620 marquant une date clé. L'orthodoxie religieuse de ceux qui croyaient impossible la réforme de l'Église anglicane les poussait, en mettant le pied en Amérique, à se concevoir comme le peuple élu.

On notera avec intérêt qu'à l'ère Reagan, autre période de messianisme intense, Updike reprend intertextuellement avec Ce que pensait Roger le grand roman fondateur des États-Unis qui exprime avec le plus de force l'esprit du puritanisme, à savoir $L a$ lettre écarlate de Nathaniel Hawthorne. Esther, comme Hester Prynne, aura un enfant d'un pasteur mais, contrairement à Dimmesdale, Lambert a déjà abandonné sa communauté avant cette naissance. Il a quitté sa première femme entre autres parce qu'elle ne pouvait lui donner d'enfant. Ce qui chez Hawthorne relève du péché tient plutôt ici de la volonté. Dale est autant torturé par sa foi que le pasteur Dimmesdale, mais pour des raisons différentes. Si, chez Hawthorne, on tente d'enlever son enfant à Hester, chez Updike c'est plutôt à Verna, par le biais de l'assistance sociale, qu'on veut retirer l'enfant (la petite Pearl, 150 ans plus tard, se nomme Paula).

Les liens sont nombreux sans limiter le roman à un simple pastiche. Je ne veux pas m'éterniser puisqu'il existe déjà de nombreux articles sur ce sujet ${ }^{28}$. Il me semble plus intéressant

27. Jean Delumeau, op. cit., p. 261.

28. Voir par exemple: Raymond Wilson, "Roger's Version: Updike's NegativeSolid Model of The Scarlet Letter", Modern Fiction Studies, vol. XXXV, n ${ }^{\circ} 2$, 1989, p. 241-250; Donald Greiner, "Body and Soul: John Updike and The Scarlet Letter", Journal of Modern Literature, vol. XV, no 4, 1989, p. 475-495. 
112

dans le contexte de cet article de constater qu'un roman habité d'un bout à l'autre, au plan diégétique, par un questionnement sur la fin, le projette également dans sa structure intertextuelle.

On a souvent dit qu'Ulysse de Joyce pouvait se lire comme un aveu d'échec, le signe que la littérature n'ayant plus rien à dire ne pouvait que se répéter. Ulysse n'est pas que répétition, mais dans un roman plus classique comme celui d'Updike, l'hypothèse d'une fin de la littérature se pose d'une manière peutêtre plus directe. Selon la formule marxiste, une tragédie qui se répète devient une farce et c'est un peu ce que l'on constate ici. La reprise d'un roman fondateur qui s'élève au rang des grandes tragédies devient avec Updike une petite comédie bourgeoise. S'il interroge avec magnificence le savoir et les limites de celui-ci quant à la quête des origines, il montre également l'ampleur de la petitesse de l'être humain. "T'es un drôle de type, Tonton. Tu veux pas baiser, tu veux pas mourir. Tu veux faire quoi?" (p. 177) demande Verna à Roger Lambert. Voilà à quoi est ramené le tragique de La lettre écarlate.

Sur le plan plus large des savoirs, Updike indique, par le biais romanesque, ce que Thomas Kuhn notamment avait démontré sur un plan sociologique et historique: une révolution scientifique entraîne toujours une mutation de sens. La fin du $\mathrm{xx}^{\mathrm{e}}$ siècle, à travers le pouvoir des ordinateurs, déplace (et déplacera, sans doute encore plus au cours des prochaines décennies) les questions. Mais Kohler affirme également, dans toute son anxiété, que la science à elle seule, par définition et par méthode, est inapte à répondre à la question du sens qui n'est pas universelle - elle ne concerne qu'une partie infime de la vie, celle des homo sapiens, jusqu'à preuve du contraire cependant qu'elle habite entièrement la conscience humaine. Kohler a tort de s'acharner à trouver des réponses. Le savoir consiste aussi à poser des questions qui n'ont pour objectif que de déplacer et de reposer les problèmes. Qu'importe que Dieu existe pour le théologien Lambert. Même "l'impossibilité du réel [...] ne constitue pas une preuve totalement originale de l'existence de Dieu" (p. 107). 